\title{
萠-环八四噻吩衍生物的合成与发光现象研究
}

\author{
杨玉杰 $a$ 徐莉*, $a$ 王华*,b \\ ( ${ }^{a}$ 河南大学化学化工学院 河南开封 475004) \\ ( ${ }^{b}$ 河南大学纳米材料工程研究中心 河南开封 475004)
}

\begin{abstract}
摘要 环八四噻吩(COTh)是一类新发现的具有聚集诱导发光(AIE)特性的 “马鞍型” 分子. 将发光基团萠引入到 COTh 分子骨架, 设计合成了三种萠-环八四噻吩化合物, 即单萠基环八四噻吩(Py-COTh)、四萠基环八四噻吩(4Py-COTh) 以及四(三(三甲硅基)环八四噻吩基)萠(12TMS-4COTh-Py). 考察了它们的吸收光谱、溶液态与冻结态(77 K)下的苂光 光谱以及聚集诱导发光(AIE)行为. 研究发现 COTh 分子骨架上萠基数量的增加对分子的共轭效应影响较小, 但其单分 子与聚集态的发光行为显著增强且发光峰位红移; 而萠基骨架上 COTh 基团数量的增加, 增加了分子的共轭效应, 造 成了吸收光谱与荧光发射光谱峰位的大范围红移. 这些光物理现象与分子结构存在着显著的构效关系.
\end{abstract}

关键词 环八四噻吩; 萠; 衍生物合成; 吸收光谱; 苂光光谱; 聚集诱导发光; 构效关系

\section{Synthesis of Pyrene-Cyclooctatetrathiophene Derivatives and Their Behaviors of Photoluminescence}

\author{
Yang, Yujie ${ }^{a} \quad \mathrm{Xu}, \mathrm{Li}^{*, a} \quad$ Wang, Hua ${ }^{*, b}$ \\ ( ${ }^{a}$ College of Chemistry and Chemical Engineering, Henan University, Kaifeng, Henan 475004) \\ $\left({ }^{b}\right.$ Research Center for Nanomaterials, Henan University, Kaifeng, Henan 475004)
}

\begin{abstract}
Cyclooctatetrathiophene (COTh) is a saddle-typed molecule with the interesting property of aggregation induced emission (AIE) discovered recently. In this paper, pyrene was introduced to the framework of COTh as a luminescent group. Three pyrene-COTh derivatives, mono-pyrene substituted COTh (Py-COTh), tetra-pyrene substituted COTh (4Py-COTh) and tetra(tri(trimethylsilyl)cyclooctatetrathiophenyl)pyrene (12TMS-4COTh-Py) were designed and synthesized. Their absorption spectra, fluorescene emission behaviors in solution, rigid state $(77 \mathrm{~K})$ and the aggregation state were explored. On the one hand, with increasing pyrene group to the framework of COTh, the molecular conjugation of pyrene-COTh derivatives, Py-COTh and 4Py-COTh changed slightly, but their emission intensity increased heavily with large red shift in both dilute solution and aggregation state. On the other hand, with the increase of COTh group to the framework of pyrene, the molecular conjugation of pyrene-COTh derivatives, Py-COTh and 12TMS-4COTh-Py changed significantly, and their emission intensity increased remarkably with large red shift in dilute solution. The relationship between the photophysical property and molecular structure is remarkably exhibited in our case.

Keywords cyclooctatetrathiophene; pyrene; synthesis of derivative; absorption spectrum; fluorescence spectrum; aggregation induced emission; structure-activity relationship
\end{abstract}

环八四噻吩是一类具有马鞍型分子构型、分子内核 为环辛四烯结构的环状四噻吩化合物. 自 1974 年 Kauffmann ${ }^{[1]}$ 首次报道以来, 有关环八四噻吩的研究工 作逐步形成了人们的研究热点 ${ }^{[2]}$. 主要包括 Marsella 报 道了以环八四噻吩为构筑模块的小分子、聚合物的合成 与分子肌肉特性研究 ${ }^{[3]}$; 笔者报道了环八四噻吩同分异 构体 ${ }^{[4]}$ 、基于环八四噻吩单元的全噻吩树枝状化合物 ${ }^{[5]}$
与双螺旋体化合物 ${ }^{[6]}$ 的构筑与分子单晶结构研究. 近期 又出现了环八四噻吩在超分子化学 ${ }^{[7]}$ 、有机太阳能电 池 ${ }^{[8]}$ 以及聚集诱导发光(AIE) $)^{[9,10]}$ 等领域的研究报道.

唐本忠等 ${ }^{[10]}$ 近日报道了环八四噻吩的基于分子内 振动受阻(RIV)机制的聚集诱导发光(AIE)现象. 然而其 AIE 的发光效率很低, 笔者考虑到芳基的引入可能会增 强芳基环八四噻吩的发光能力, 特别是萠基具有优异的

* Corresponding authors. E-mail: xuli@henu.edu.cn; hwang@henu.edu.cn

Received December 11, 2019; revised February 29, 2020; published online March 6, 2020.

Project supported by the National Natural Science Foundation of China (Nos. 21672053, 21672054 ).

国家自然科学基金(Nos. 21672053, 21672053)、河南省有机功能材料创新团队(No. C20150011)资助项目. 
发光能力与稳定性, 常作为荧光探针用于光物理领域的 研究. 基于这方面的考虑, 为此本文设计合成了三种分 子结构特色显著的萠-环八四噻吩化合物, 即单萠基环 八四噻吩 $(\mathbf{P y}-\mathrm{COTh}) 、$ 四萠基环八四噻吩(4Py-COTh) 以及四 (三 (三甲硅基)-环八四噻吩基)萠(12TMS4COTh-Py). 通过考察这三种化合物的光物理行为, 特 别是苂光与 AIE 行为, 研究它们的分子结构的差异对光 物理行为的影响, 得到了一些有趣的光物理现象并明确 了相应的构效关系.

\section{1 结果与讨论}

\section{1 化合物的合成}

目标化合物的合成路线如图 1 所示, 以笔者课题组 报道过的化合物 4TMS-COTh ${ }^{[11]}$ 为中间体, 用稀释的三 氟乙酸选择性地脱去 4TMS-COTh 分子中的一个三甲 硅基(TMS)基团制备 3TMS-COTh ${ }^{[4]}$. 3TMS-COTh 是个 重要的中间体, 一方面用二异丙基氨基锂(LDA)夺其噻 吩环上 $\alpha$-位质子产生碳负离子, 再用 $\mathrm{C}_{2} \mathrm{Cl}_{4} \mathrm{Br}_{2}$ 对所形成 的碳负离子进行淬灭溴代, 合成 Br-3TMS-COTh ${ }^{[12]}$. 其后与芘基硼酸通过 Suzuki 偶联反应合成 Py-3TMS-COTh, 最后经三氟乙酸脱去 TMS 基团, 得 到目标化合物 Py-COTh. 另一方面, 通过 $n$-BuLi 夺 3TMS-COTh 噻吩环上 $\alpha$-位质子, 所形成的碳负离子与 2-异丙氧基-4,4,5,5-四甲基- 1,3,2-二氧环戊嗍烷反应制 备环八四噻吩的硓酸酯 $(\text { B-3TMS-COTh })^{[7 \mathrm{a}]}$, 其后与 1,3,6,8-四溴芘通过 Suzuki 偶联反应合成目标化合物 12TMS-4COTh-Py. 化合物 4TMS-COTh 通过 $N$-溴代 丁二酰亚胺(NBS) 在乙酸中直接四溴代, 四溴代化合物 $4 \mathrm{Br}-\mathrm{COTh}^{[5]}$ 与 1-萠嗍酸通过 Suzuki 偶联反应获得目标 化合物 4Py-COTh.

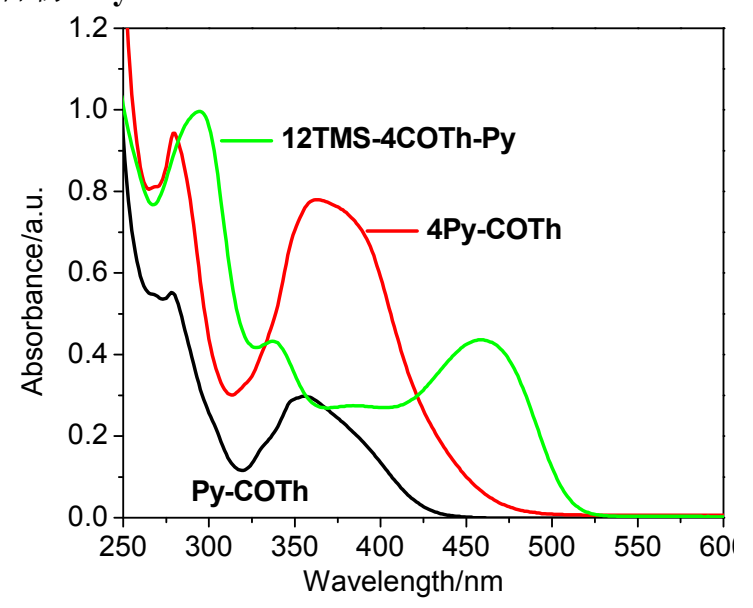

图 1 化合物 Py-COTh、4Py-COTh 与 12TMS-4COTh-Py 在 四氢呋喃中的吸收光谱 $\left(c=1 \times 10^{-5} \mathrm{~mol} / \mathrm{L}\right)$

Figure 1 UV-vis absorption spectra of Py-COTh, 4Py-COTh and 12TMS-4COTh-Py in tetrahydrofuran (THF) $\left(c=1 \times 10^{-5}\right.$ $\mathrm{mol} / \mathrm{L}$ )
以上三个目标化合物均通过了核磁、红外以及高分 辨质谱的鉴定确认了它们的分子结构.

\section{2 化合物的光谱性质}

\subsection{1 吸收光谱}

图 1 给出了三个目标化合物的紫外可见吸收光谱, 包含 $280 \sim 300 \mathrm{~nm}$ 的环八四噻吩(COTh) 骨架的吸收 ${ }^{[5]}$ 与 $320 \sim 420 \mathrm{~nm}$ 范围的萠基与相连噻吩环之间的共轭吸 收; 与 Py-COTh 在 $357 \mathrm{~nm}$ 处的吸收峰相比, 萠基数量 的增加, 造成了 $4 \mathrm{Py}-\mathrm{COTh}$ 的长波长吸收峰的强度大大 增加, 但其峰位 $(363 \mathrm{~nm}$ ) 只红移了 $6 \mathrm{~nm}$, 说明多个萠基 的引入对整个分子的共轭效应的贡献有限不大. $4 \mathbf{P y}-$ COTh 的长波吸收峰强度相当于四个 Py-COTh 的长波 吸收峰强度的加和, 这一现象是由环八四噻吩结构单元 自身的交叉共轭的特点决定的 ${ }^{[5]}$. 然而 12TMS-4COTh-Py 表现出非常显著的吸收峰位的红移, 最大吸收峰 在 $458 \mathrm{~nm}$ 处, 与 Py-COTh 在 $357 \mathrm{~nm}$ 处的吸收峰相比 红移了 $101 \mathrm{~nm}$, 说明作为给电子基团的四个环八四噻 吩单元与中心萠单元存在较好的共轭, 拓展了以平面萠 为中心的分子整体的共轭效应，从而造成吸收峰位的大 范围红移.

\subsection{2 荧光光谱}

图 2 给出了化合物 Py-COTh、4Py-COTh 与 12 TMS-4COTh-Py 在四氢呋喃中于室温(图 2a) 和 $77 \mathrm{~K}$ (图 $2 \mathrm{~b})$ 条件下的苂光光谱. 从室温苂光可以看出, Py-COTh 在 400 480 nm 范围发比较微弱的苂光; 4Py-COTh 除 了短波长的荧光发射外, 还在长波长范围有较强的发 光, 峰位在 $573 \mathrm{~nm}$. 其发光寿命为 $1.34 \mathrm{~ns}$, 说明该发光 是苂光, 而不是磷光. 由于 $4 \mathrm{Py}-\mathrm{COTh}$ 分子结构中存在 四个萠基, 而萠基又是平面性很好的基团，与相连的噻 吩环之间呈近平面构象 ${ }^{[5]}$. 这就造成了一方面分子自身 的共轭性较好，另一方面激发态下可能形成分子间的类 似激基缔合物的相互作用，从而产生长波长的发射. 12TMS-4COTh-Py 的发光峰位在 $523 \mathrm{~nm}$, 尽管从吸收 光谱中可以看出, 该分子具有最强的分子内的共轭效 应, 但由于 COTh 单元的非平面性，与萠基相连的四个 COTh 单元各自又有三个 TMS 大位阻基团, 造成该分 子在激发态下分子间无法存在近距离的相互作用, 与 4Py-COTh 分子的发光行为相比, 12TMS-4COTh-COTh 表现短波长的荧光发射峰, 表现出单分子自身发光 特征. 与 COTh 的发光能力 (荧光量子产率 $0.4 \%$ ) 相 比 $^{[10]}$, Py-COTh、4Py-COTh 与 12TMS-4COTh-Py 在 四氢呋喃中的荧光量子产率分别为 $0.8 \% 、 1.8 \%$ 与 $10.0 \%$ (表 1), 说明萠基的引入的确增强了分子的整体发光能 力.

冻结态是研究单分子发光行为的理想状态, 因为在 
<smiles>CS(=O)(=O)c1cc2c(s1)-c1sc(Br)cc1-c1cc(S(C)(=O)=O)sc1-c1sc(S(C)(=O)=O)cc1-2</smiles>

Br-3TMS-COTh
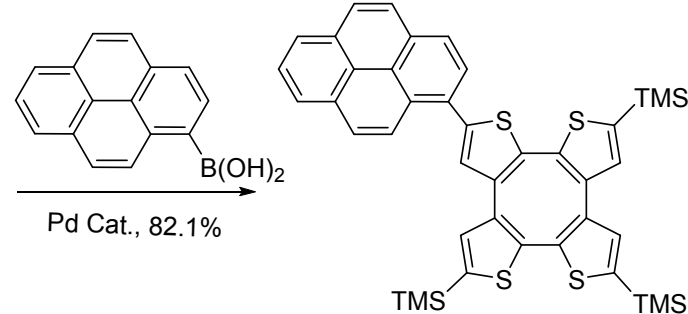

Py-3TMS-COTh

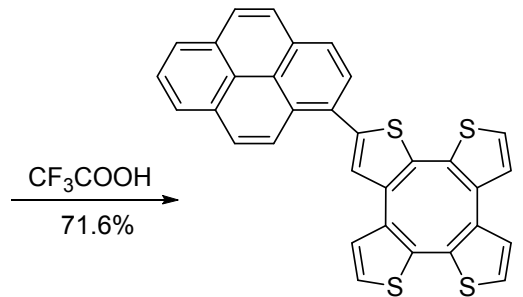

Py-COTh

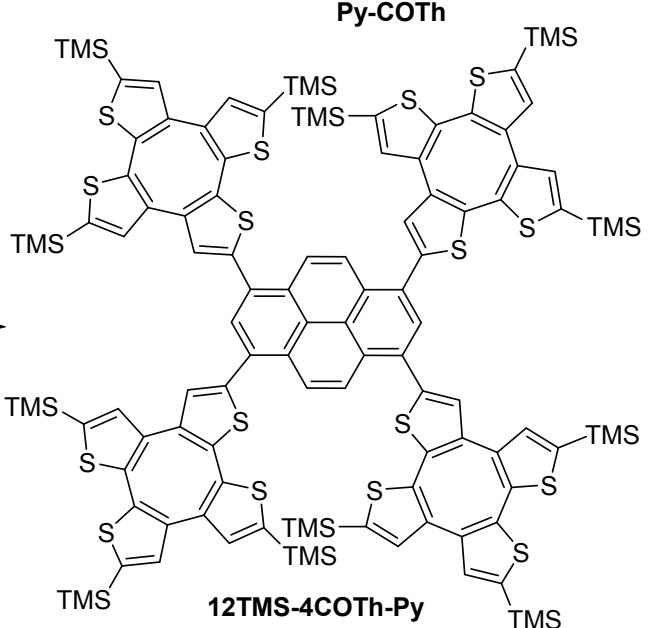
$\mathrm{CF}_{3} \mathrm{COOH}$

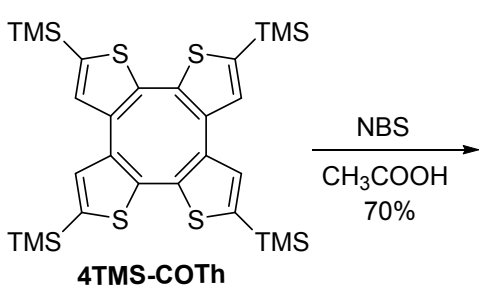
4TMS-COTh<smiles>Brc1cc2c(s1)-c1cc(Br)sc1-c1cc(Br)sc1-c1sc(Br)cc1-2</smiles><smiles>Oc1ccc2ccc3cccc4ccc1c2c34</smiles>

Pd. cat, $81.9 \%$

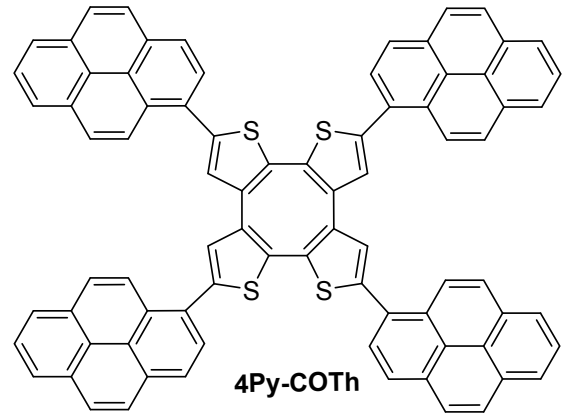

图式 1 萠-环八四噻吩衍生物的合成路线

Scheme 1 Synthetic route to pyrene-COTh derivatives

表 1 化合物 Py-COTh、4Py-COTh、12TMS-4COTh-Py 的 光谱数据 ${ }^{a}$

Table 1 Spectroscopic data for Py-COTh, 4Py-COTh and 12TMS-4COTh-Py

\begin{tabular}{|c|c|c|c|c|c|c|}
\hline Compd. & $\lambda_{\mathrm{ab}} / \mathrm{nm}$ & $\begin{array}{l}\text { PL RT } \\
\lambda_{\mathrm{em}} / \mathrm{nm}\end{array}$ & $\mathrm{QY}^{* /}$ & $\tau / \mathrm{ns}$ & $\begin{array}{c}\text { PL } 77 \mathrm{~K} \\
\lambda_{\mathrm{em}} / \mathrm{nm}\end{array}$ & $\begin{array}{c}\text { AIE } \\
\lambda_{\mathrm{em}} / \mathrm{nm}\end{array}$ \\
\hline & & 432 & 0.8 & 1.75 & 488 & 515 \\
\hline 4Py-COTh & & 573 & 1.8 & 1.34 & 498 & 542 \\
\hline 12TMS-4COTh-Py & 458 & 523 & 10.0 & 0.89 & 504 & 520 \\
\hline
\end{tabular}

${ }^{a}$ Relative fluorescence quantum yield (QY) determined by quinine sulfate (in $\left.1 \mathrm{~mol} \cdot \mathrm{L}^{-1} \mathrm{H}_{2} \mathrm{SO}_{4}\right)$ as a reference $(\mathrm{QY}=0.55)^{[13]}$.

冻结态下, 分子间的运动、分子内的振动以及溶剂分子 的动态溶剂化作用均消失, 多种非辐射失活途径被遏 制, 分子的发光强度通常将大幅度增加, 并伴随着发光 峰位的蓝移. 图 $2 \mathrm{~b}$ 图给出了 $77 \mathrm{~K}$ 条件下化合物的苂光 光谱. 不同于室温苂光行为, 三者除了出现较弱的短波 长的本征态发光外, 在 $450 \sim 570 \mathrm{~nm}$ 范围内均出现了长 波长的苂光发射. 其中, Py-COTh 分子发射了峰位位于
$488 \mathrm{~nm}$ 的分子共轭体系的发光. 由于失去分子间的相 互作用、溶剂化作用, 特别是环八四噻吩骨架构型翻转 过程的冻结限制了分子动态共轭效应的形成, 4PyCOTh 分子自身的发光峰位大范围蓝移至 $498 \mathrm{~nm}$. 而分 子共轭效应最好的 12TMS-4COTh-Py 分子发光峰位蓝 移至 $504 \mathrm{~nm}$, 发射光谱展示出一定的精细结构(534 nm 处出现肩峰). 三者的发光峰位(表 1)与它们的分子自身 的共轭程度相一致.

\subsubsection{AIE 荧光光谱}

我们考察了 Py-COTh、4Py-COTh 与 12TMS-4COThPy 在四氢呋喃-水二元体系中的 AIE 性质, 发现 Py-COTh 与 4Py-COTh 表现出显著的 AIE 特性, 而 12TMS-4COTh-Py 没有展示出明显的 AIE 现象. Py-COTh 与 4Py-COTh 分子尺寸不同, 当水的体积分 数大于 $70 \%$ 时, 由于疏水作用造成分子聚集 ${ }^{[14],} \mathbf{P y}-$ COTh 在 $515 \mathrm{~nm}$ 处表现出显著的 AIE 发光特性; 当体 

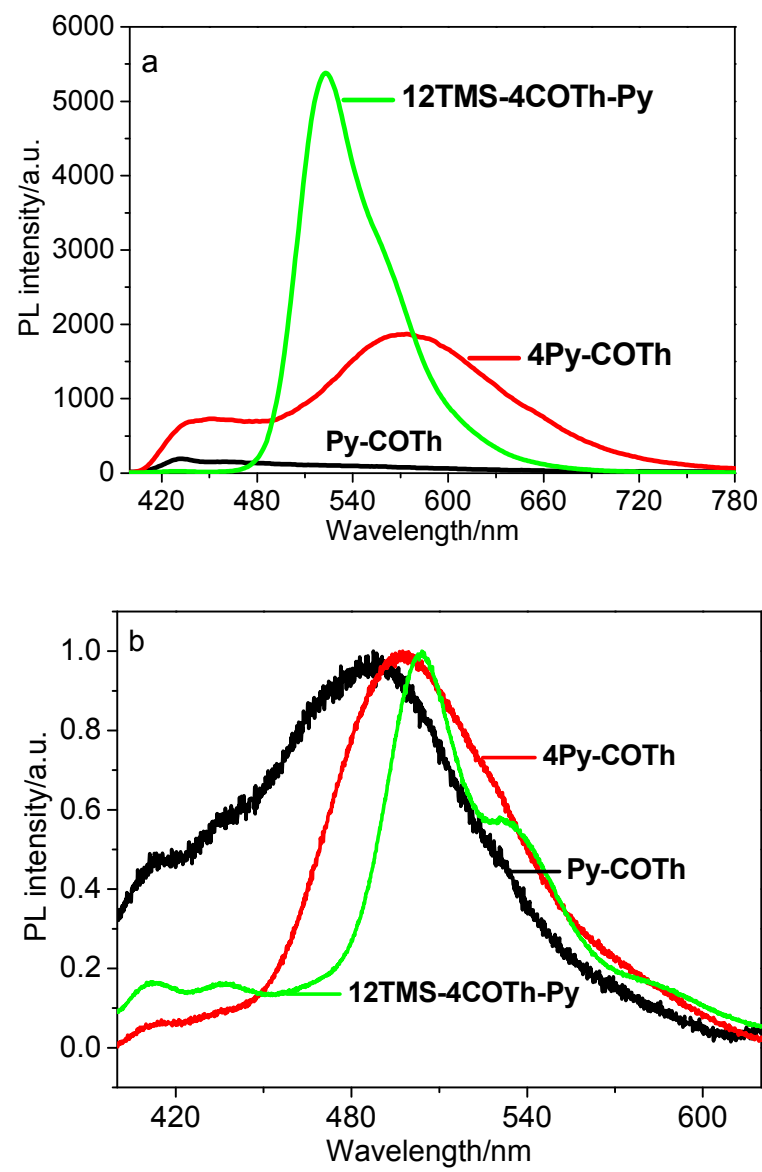

图 2 化合物 Py-COTh、4Py-COTh 与 12TMS-4COTh-Py 在

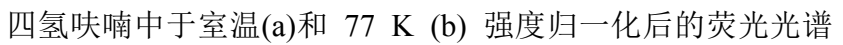
$\left(c=1 \times 10^{-5} \mathrm{~mol} / \mathrm{L}\right)$

Figure 2 Photoluminence (PL) spectra of Py-COTh, 4PyCOTh and 12TMS- 4COTh-Py in THF at room temperature (a) and $77 \mathrm{~K}$ (b) normalized spectra $\left(c=1 \times 10^{-5} \mathrm{~mol} / \mathrm{L}, \lambda_{\mathrm{ex}}=380\right.$ $\mathrm{nm})$

积分数为 $90 \%$ 时, AIE 的发光强度最大(图 3). 分子尺寸 较大的 4Py-COTh 分子具有更强的疏水作用. 体积分数 为 $40 \%$ 时, 就出现 AIE 发光现象, 发光峰位为 $542 \mathrm{~nm}$. 当体积分数为 $70 \%$ 时, AIE 的发光强度就达到最大值(图 4).

Py-COTh 与 4Py-COTh 表现出的 AIE 现象与分子 间的紧密聚集密切相关. 由于平面性非常好的萠基团的 存在, 分子间聚集时萠基团之间的 $\pi-\pi$ 相互作用可以加 强分子间的紧密聚集, 同时环八四噻吩之间也存在着显

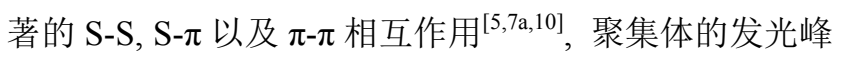
位大幅度红移. 与两个萠基之间形成的激基缔合物的发 射峰在 $480 \mathrm{~nm}$ 相比 ${ }^{[15]}, 515 \mathrm{~nm}$ 的 AIE 发光峰位显示了 激发态下分子聚集体对激发态能量的分散. 拥有四个萠 基的 4Py-COTh 在分子聚集时, 分子间的相互作用更 强, 其 AIE 发光强度增加, 发光峰位由溶液态的 $573 \mathrm{~nm}$ 蓝移至聚集态的 $542 \mathrm{~nm}$. 可能与溶剂化作用的大幅消
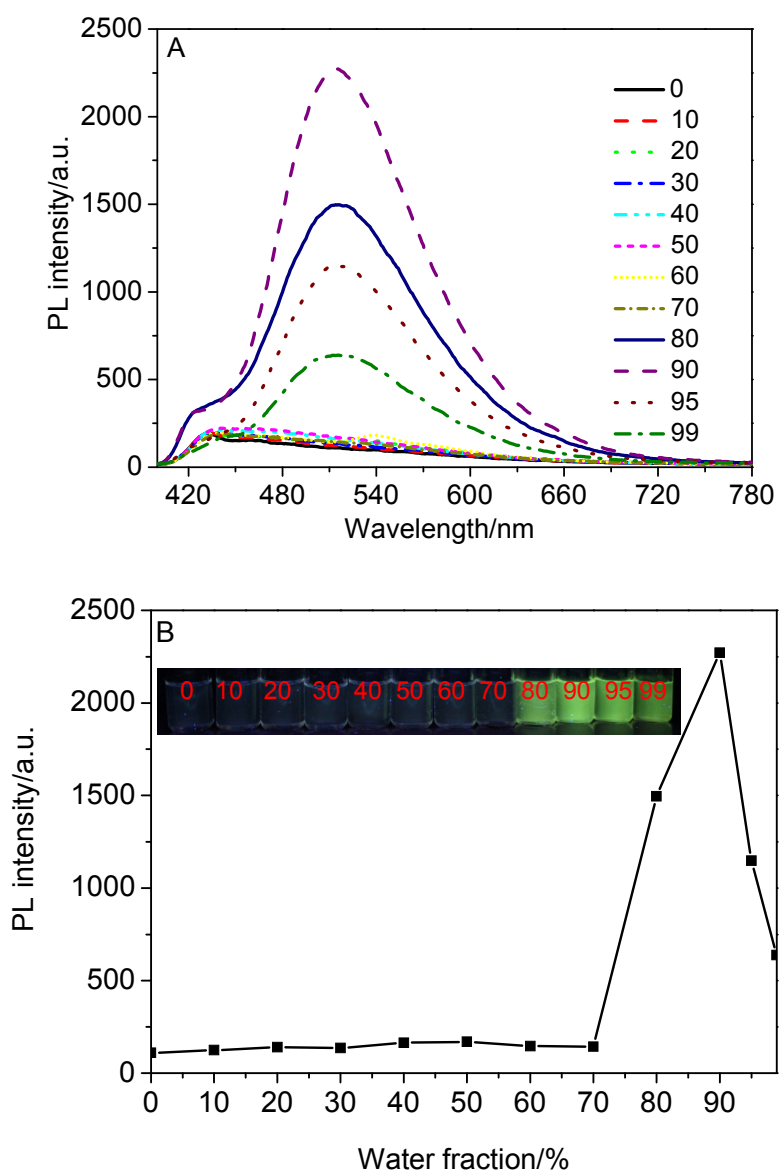

图 3 (A) Py-COTh 在 THF/水混合溶剂中的苂光光谱 $(c=1 \times$ $10^{-5} \mathrm{~mol} / \mathrm{L}, \lambda_{\mathrm{ex}}=380 \mathrm{~nm}$ ) 和(B) $515 \mathrm{~nm}$ 处发光强度与水的体积 分数之间的关系图 $(365 \mathrm{~nm})$

Figure 3 (A) PL spectra of Py-COTh in THF/water mixtures with different water fractions $\left(c=1 \times 10^{-5} \mathrm{~mol} / \mathrm{L}, \lambda_{\mathrm{ex}}=380 \mathrm{~nm}\right)$, and (B) the changes of PL peak intensities at $515 \mathrm{~nm}$ versus the water fraction in the THF/water mixtures

Inset: Fluorescence photographs under the illumination of a UV lamp $(365 \mathrm{~nm})$

退以及形成 $\mathrm{H}$-聚集体有关 ${ }^{[16]}$.

而 12TMS-4COTh-Py 分子由于其四周为 4 个非平 面的 “马鞍型” 环八四噻吩以及 12 个大位阻的 TMS 基 团，不利于分子间的紧密聚集. 因此在不良溶剂中， 12TMS-4COTh-Py 分子没有出现显著的分子聚集体的 发光现象(图 5)以及发射峰的红移或蓝移现象, 仅表现 出单分子自身的发光行为. 说明在不良溶剂中的分子疏 水作用下, 12TMS-4COTh-Py 分子无法形成有效的分子 间紧密聚集.

从以上实验可以看出, Py-COTh、4Py-COTh 与 12TMS-4COTh-Py 在不良溶剂中的发光行为与它们的 分子结构存在显著的构效关系.

\section{2 结论}

环八四噻吩 $($ COTh) 是一类新型的基于分子振动受 

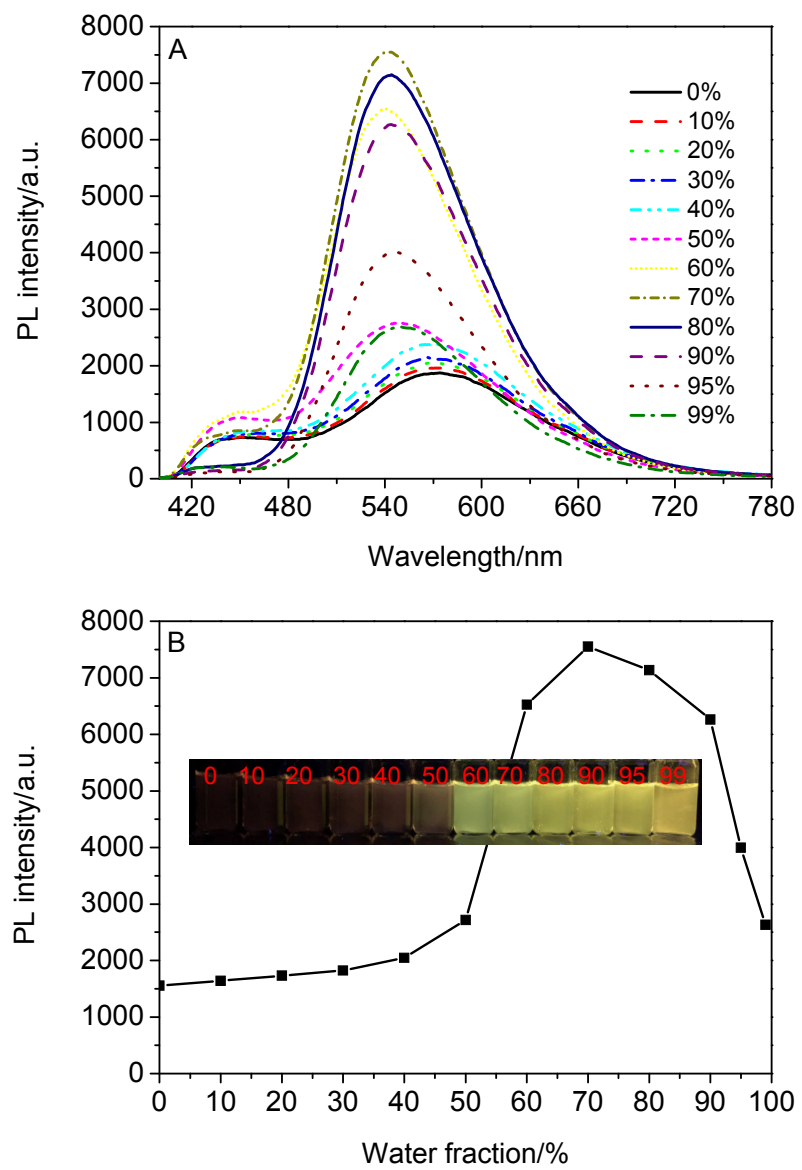

图 4 (A) 4Py-COTh 在 $\mathrm{THF} /$ 水混合溶剂中的苂光光谱 $(c=$ $1 \times 10^{-5} \mathrm{~mol} / \mathrm{L}, \lambda_{\mathrm{ex}}=380 \mathrm{~nm}$ )和(B) $542 \mathrm{~nm}$ 处发光强度与水的 体积分数之间的关系图

Figure 4 (A) PL spectra of 4Py-COTh in THF/water mixtures with different water fractions $\left(c=1 \times 10^{-5} \mathrm{~mol} / \mathrm{L}, \lambda_{\mathrm{ex}}=380 \mathrm{~nm}\right)$, and the changes of PL peak intensities at $542 \mathrm{~nm}$ versus the water fraction in the THF/water mixtures

Inset: Fluorescence photographs under the illumination of a UV lamp (365 nm)

阻(RIV)机制的聚集诱导发光(AIE)新结构分子. 基于发 光增强的考虑, 本文通过合成设计, 制备了三种分子结 构新颖、富有功能的萠-环八四噻吩化合物, 即 $\mathbf{P y}-$ COTh、4Py-COTh 和 12TMS-4COTh-Py. 通过考察它 们的吸收光谱、室温与冻结态下的苂光光谱以及 AIE 发 光行为, 研究发现萠基的引入的确可以显著增强分子整 体的发光能力. 一方面, COTh 分子骨架上萠基数量的 增加对分子的共轭效应影响较小. 如 Py-COTh 与 4PyCOTh 相比, 吸收峰位红移不大, 但发光行为强度随萠 基数量增加而增强, 且发光峰位显著红移, 其中 AIE 发 光能力大幅增强, 表现出显著的芳基取代基效应与疏水 效应. 另一方面, 萠基骨架上 COTh 基团数量的增加可 以对分子的共轭效应影响较大. 如Py-COTh 与 12TMS4COTh-Py 相比, 后者的吸收光谱大范围红移, 发光行 为随 COTh 基团数量增加而显著红移, 表现出显著的
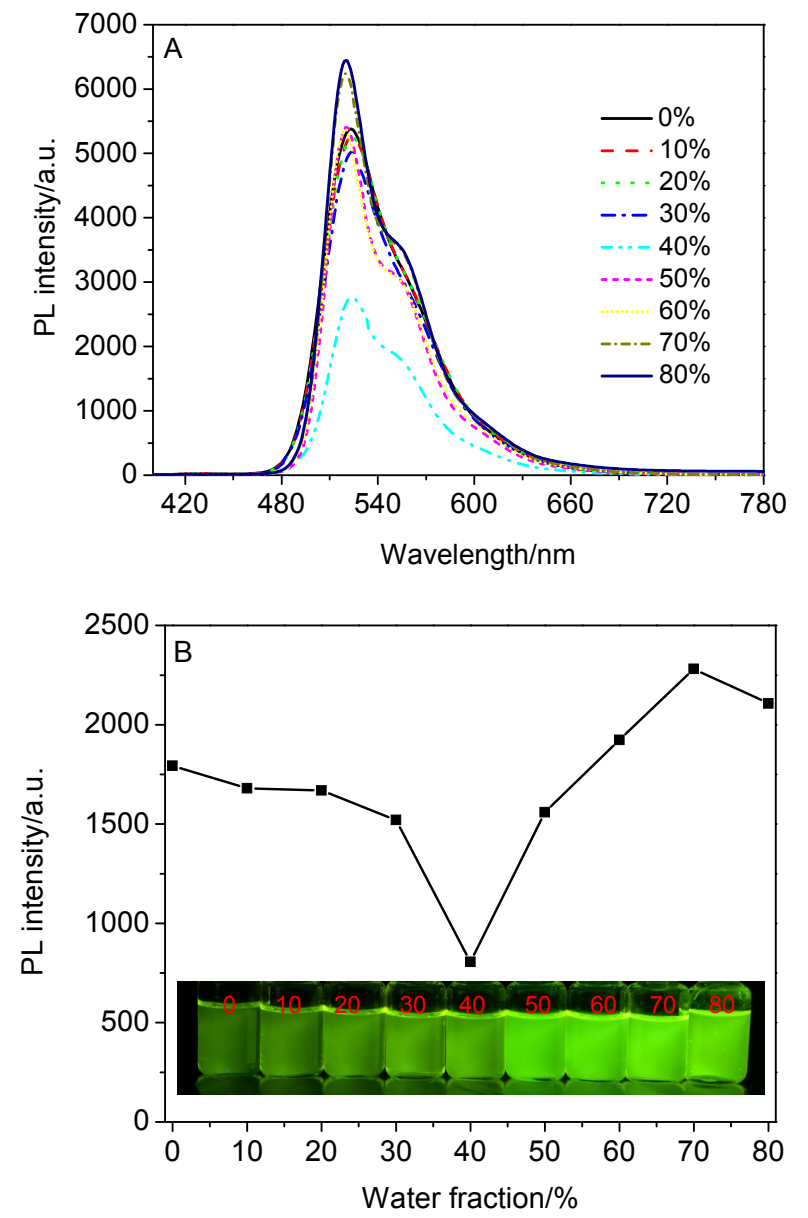

图 5 (A) 12TMS-4COTh-Py 在 THF/水混合溶剂中的苂光光 谱 $\left(c=1 \times 10^{-5} \mathrm{~mol} / \mathrm{L}, \lambda_{\mathrm{ex}}=380 \mathrm{~nm}\right.$ )和(B) $520 \mathrm{~nm}$ 处发光强度 与水的体积分数之间的关系图

Figure 5 (A) PL spectra of 12TMS-4COTh-Py in THF/water mixtures with different water fractions $\left(c=1 \times 10^{-5} \mathrm{~mol} / \mathrm{L}, \lambda_{\mathrm{ex}}=\right.$ $380 \mathrm{~nm}$ ), and (B) the changes of PL peak intensities at $520 \mathrm{~nm}$ versus the water fraction in the $\mathrm{THF} /$ water mixtures Inset: Fluorescence photographs under the illumination of a UV lamp (365 nm).

取代基的共轭效应. 分子间聚集能力较差的 12 TMS-4COTh-Py 则不能表现出显著的 AIE 发光现象. 这些分子结构与发光现象之间显著构效关系的揭示，将 拓展基于环八四噻吩这类新型 AIE 分子材料结构的多 样性, 为人们设计新型发光材料提供理论基础与应用素 材.

\section{3 实验部分}

\section{1 仪器与试剂}

核磁共振谱仪(AVANCE $400 \mathrm{MHz}$, Bruker), 高分辨 质谱仪(Thermo Scientific LTQ Orbitrap XL), 稳态-瞬态 荧光光谱仪(JY HORIBA FluoroLog-3)或上海棱光 F97, 红外光谱仪(VERTEX 70, Bruker), 紫外可见分光光度 计(UV-1900, Shimadzu), X 射线单晶衍射仪(Apex II, 
Bruker-AXS, Bruker), 显微熔点测定仪(TX4-100).

实验试剂 1-萠硼酸、四(三苯基膦)钯、1,3,6,8-四溴 萠来自安耐吉产品. 4TMS-COTh ${ }^{[10]}, 3 \mathbf{T M S}-\mathrm{COTh}^{[5]}$, Br-3TMS-COTh ${ }^{[12]}$, 4Br-COTh ${ }^{[5]}$, B-3TMS-COTh ${ }^{[7 a]}$ 按 照文献方法制备.

\section{2 化合物 Py-COTh、4Py-COTh 和 12TMS-4COTh- $\mathrm{Py}$ 的合成}

\subsection{1 化合物 Py-3TMS-COTh 的合成}

将 Br-3TMS-COTh (120.2 mg, $0.18 \mathrm{mmol}, 1.0$ equiv.)、1-萠硼酸(55.7 mg, $0.23 \mathrm{mmol}, 1.3$ equiv.)、无水 碳酸钾(62.2 $\mathrm{mg}, 0.45 \mathrm{mmol}, 2.5$ equiv.)和四(三苯基膦) 钯(13.2 mg, $0.009 \mathrm{mmol}, 0.2$ equiv.)加入 $50 \mathrm{~mL}$ Schlenk 瓶中. 氩气保护下, 加入四氢呋喃 $(20 \mathrm{~mL})$ 和无氧水 $(2$ $\mathrm{mL}), 85{ }^{\circ} \mathrm{C}$ 油浴中反应过夜. 减压旋转反应溶剂, 二氯 甲烷 $(10 \mathrm{~mL} \times 3)$ 萃取, 蒸馏水 $(30 \mathrm{~mL})$ 洗涤, 有机相经无 水硫酸镁干燥, 过滤、旋蒸后的粗品经品柱层析 [300 400 目硅胶, 淋洗剂: $V$ (石油醚) $: V$ (二氯甲烷 $)=7 ： 1]$, 再用三氯甲烷-甲醇 $(V: V=1: 3)$ 进行重结晶, 获得黄 色固体 Py-3TMS-COTh $117.9 \mathrm{mg}$, 产率 82.1\%. m.p. 290 $292{ }^{\circ} \mathrm{C} ;{ }^{1} \mathrm{H}$ NMR (400 MHz, $\left.\mathrm{CDCl}_{3}\right) \delta: 8.68$ (d, $J=$ $9.2 \mathrm{~Hz}, 1 \mathrm{H}), 8.22 \sim 8.18(\mathrm{~m}, 3 \mathrm{H}), 8.14(\mathrm{~s}, 1 \mathrm{H}), 8.12(\mathrm{~s}$, $1 \mathrm{H}), 8.10(\mathrm{~s}, 1 \mathrm{H}), 8.09(\mathrm{~s}, 1 \mathrm{H}), 8.07 \sim 7.99(\mathrm{~m}, 1 \mathrm{H}), 7.31$ $(\mathrm{s}, 1 \mathrm{H}), 7.15(\mathrm{~s}, 1 \mathrm{H}), 7.13(\mathrm{~s}, 1 \mathrm{H}), 0.38(\mathrm{~s}, 9 \mathrm{H}), 0.37$ (s, 9H), 0.32 (s, 9H); ${ }^{13} \mathrm{C}$ NMR (100 MHz, $\left.\mathrm{CDCl}_{3}\right) \delta: 143.91$, $142.80,142.76,142.73,138.20,137.99,137.87,137.56$, $137.54,137.54,137.49,137.07,136.93,136.63,133.24$, $131.56,131.24,131.07,130.86,129.21,128.85,128.21$, $128.01,127.43,126.29,125.52,125.24,125.22,125.12$, 124.88, 124.81, 100.08, 0.01, -0.01, - 0.07; IR (KBr) v: 2858, $838 \mathrm{~cm}^{-1}$. HRMS (ESI) calcd for $\mathrm{C}_{41} \mathrm{H}_{41} \mathrm{~S}_{4} \mathrm{Si}_{3}$ 745.1393, found 745.1393.

\subsection{2 化合物 Py-COTh 的合成}

将 Py-3TMS-COTh (117.9 mg, $0.16 \mathrm{mmol}, 1.0$ equiv.)放入 $100 \mathrm{~mL}$ 圆底烧瓶中, 加入三氯甲烷 $(15 \mathrm{~mL})$ 使之完全溶解, 快速摚拌下滴加 $\mathrm{CF}_{3} \mathrm{COOH}(1.0 \mathrm{~mL}$, 过 量), 薄层色谱跟踪反应, 反应约 $0.5 \mathrm{~h}$ 结束. 小心加入 饱和碳酸氢钠淬灭反应至弱碱性, 用二氯甲烷 $(20 \mathrm{~mL} \times$ 3)萃取, 无水硫酸镁干燥, 经过滤、旋蒸后的粗品经柱 层析 $[300 \sim 400$ 目硅胶, 淋洗剂: $V$ (石油醚)： $V$ (二氯甲 烷 $)=7: 1]$, 再用三氯甲烷-甲醇 $(V: V=1: 4)$ 进行沉 降, 获得黄色固体 Py-COTh 80.5 mg, 产率 96.3\%. m.p. 235 237 ${ }^{\circ} \mathrm{C} ;{ }^{1} \mathrm{H}$ NMR (400 MHz, $\left.\mathrm{CDCl}_{3}\right) \delta: 8.65(\mathrm{~d}, J=$ $8 \mathrm{~Hz}, 1 \mathrm{H}), 8.24 \sim 8.16(\mathrm{~m}, 3 \mathrm{H}), 8.16 \sim 8.07(\mathrm{~m}, 4 \mathrm{H})$, $8.07 \sim 7.99(\mathrm{~m}, 1 \mathrm{H}), 7.48 \sim 7.39(\mathrm{~m}, 3 \mathrm{H}), 7.27(\mathrm{~s}, 1 \mathrm{H})$, $7.07(\mathrm{~d}, J=9.2 \mathrm{~Hz}, 1 \mathrm{H}), 7.06 \sim 6.99(\mathrm{~m}, 2 \mathrm{H}) ;{ }^{13} \mathrm{C} \mathrm{NMR}$ $\left(100 \mathrm{MHz}, \mathrm{CDCl}_{3}\right) \delta: 136.64,131.42,131.17,130.48$, $130.08,130.00,128.90,128.76,128.15,128.08,127.95$, $127.30,127.23,126.20,125.45,125.17,125.07,124.89$, 124.73, 124.68; IR (KBr) v: 2924, 2853, $841 \mathrm{~cm}^{-1}$. HRMS $\left(\mathrm{EI}^{+}\right)$calcd for $\mathrm{C}_{32} \mathrm{H}_{16} \mathrm{~S}_{4} 528.0135$, found 528.0133.

\subsection{3 化合物 4Py-COTh 的合成}

将 4Br-COTh (60.0 mg, 0.093 mmol, 1.0 equiv.)、1萠硼酸(114.4 mg, $0.465 \mathrm{mmol}, 5.0$ equiv.)、无水碳酸钾 (38.4 mg, $0.279 \mathrm{mmol}, 3$ equiv.)和四(三苯基膦)钯(27.2 $\mathrm{mg}, 0.0186 \mathrm{mmol}, 0.2$ equiv.)加入 $100 \mathrm{~mL}$ Schlenk 瓶中. 氩气保护下再加入四氢呋喃 $(30 \mathrm{~mL})$ 和无氧水 $(3 \mathrm{~mL})$. 将 Schlenk 反应瓶在 $90{ }^{\circ} \mathrm{C}$ 油浴中反应过夜. 减压旋蒸 反应溶剂, 二氯甲烷 $(10 \mathrm{~mL} \times 3)$ 萃取, 蒸馏水 $(30 \mathrm{~mL})$ 洗 涤, 有机相经无水硫酸镁干燥, 旋蒸溶剂后得到粗品. 粗品用 $V$ (石油醚) $: V($ 二氯甲烷 $)=8: 1$ 进行索式提取, 获得橙色固体 4Py-COTh $86.2 \mathrm{mg}$, 产率 $81.9 \%$. m.p.> $300{ }^{\circ} \mathrm{C} ;{ }^{1} \mathrm{H}$ NMR (400 MHz, $\left.\mathrm{CDCl}_{3}\right) \delta: 8.82$ (d, $J=9.6$ $\mathrm{Hz}, 1 \mathrm{H}), 8.25$ (s, 1H), $8.23 \sim 8.17(\mathrm{~m}, 1 \mathrm{H}), 8.12$ (d, $J=2.4$ $\mathrm{Hz}, 1 \mathrm{H}), 8.03$ (t, $J=7.6 \mathrm{~Hz}, 1 \mathrm{H}), 7.54(\mathrm{~s}, 1 \mathrm{H})$; IR (KBr) v: $3444,3037,839 \mathrm{~cm}^{-1}$. HRMS $\left(\mathrm{EI}^{+}\right)$calcd for $\mathrm{C}_{80} \mathrm{H}_{40} \mathrm{~S}_{4}$ 1128.2013, found 1128.2034.

\subsection{4 化合物 12TMS-4COTh-Py 的合成}

将 B-3TMS-COTh (233.2 mg, $0.37 \mathrm{mmol}, 4.5$ equiv.)、1,3,6,8-四溴萠(40 mg, $0.08 \mathrm{mmol}, 1.0$ equiv.)、无 水碳酸钾(33.2 mg, $0.24 \mathrm{mmol}, 3$ equiv.)和四(三苯基膦) 钯(23.4 mg, $0.016 \mathrm{mmol}, 0.2$ equiv.)加入 $100 \mathrm{~mL}$ Schlenk 瓶中. 氩气保护下再加入四氢呋喃 $(40 \mathrm{~mL})$ 和无氧水 $(5$ $\mathrm{mL})$. 将 Schlenk 反应瓶在 $100{ }^{\circ} \mathrm{C}$ 油浴中反应过夜. 减 压旋蒸反应溶剂，二氯甲烷 $(15 \mathrm{~mL} \times 3)$ 萃取，蒸馏水 $(30$ $\mathrm{mL})$ 洗涤, 有机相经无水硫酸镁干燥, 旋蒸溶剂后得到 粗品. 粗品用石油醚-二氯甲烷 $(V: V=1: 3)$ 进行索式 提取, 获得橙色固体 12TMS-4COTh-Py $150.5 \mathrm{mg}$, 产 率 81.5\%. m.p. $>300{ }^{\circ} \mathrm{C} ;{ }^{1} \mathrm{H} \mathrm{NMR}\left(400 \mathrm{MHz}, \mathrm{CDCl}_{3}\right) \delta$ : $8.76 \sim 8.72(\mathrm{~m}, 4 \mathrm{H}), 8.30(\mathrm{~s}, 1 \mathrm{H}), 8.28(\mathrm{t}, J=2.4 \mathrm{~Hz}, 1 \mathrm{H})$, $7.37 \sim 7.28(\mathrm{~m}, 4 \mathrm{H}), 7.16 \sim 7.06(\mathrm{~m}, 12 \mathrm{H}), 0.40 \sim 0.26(\mathrm{~m}$, 108H). HRMS (EI ${ }^{+}$) calcd for $\mathrm{C}_{80} \mathrm{H}_{40} \mathrm{~S}_{4} 2374.2960$, found 2373.2866 .

辅助材料 (Supporting Information) Py-COTh, Py3TMS-COTh, 4Py-COTh, 12TMS-4COTh-Py 的 ${ }^{1} \mathrm{H}$ $\mathrm{NMR},{ }^{13} \mathrm{C}$ NMR, HRMS, IR 谱图. 这些材料可以免费从 本刊网站(http://sioc-journal.cn/)上下载.

\section{References}

[1] (a) Greving, B.; Woltermann, A.; Kauffmann, T. Angew. Chem., Int. Ed. 1974, 13, 467. 
(b) Kauffmann, T.; Greving, B.; König, J.; Mitschker, A.; Woltermann, A. Angew. Chem., Int. Ed. 1975, 14, 713.

[2] (a) Zhao, C.; Xu, L.; Wang, Y.; Li, C.; Wang, H. Chin. J. Chem. 2015, 33, 71 .

(b) Li, L.; Zhao, C.; Wang, H. Chem. Rec. 2016, 16, 797.

[3] Marsella, M. J.; Reid, R. J. Macromolecules 1999, 32, 5982.

[4] (a) Li, L.; Zhao, C.; Wang, H. Chem. Rec. 2016, 16, 797.

(b) Zhao, C.; Xu, L.; Wang, Y.; Li, C.; Wang, H. Chin. J. Chem. 2015, 33, 71 .

(c) Wang, Y.; Song, J.; Xu, L.; Kan, Y.; Shi, J.; Wang, H. J. Org. Chem. 2014, 79, 2255.

[5] Wang, Y.; Gao, D.; Shi, J.; Kan, Y. Song, J.; Li, C.; Wang, H. Tetrahedron 2014, 70, 631.

[6] Zhang, S.; Liu, X.; Li, C.; Li, L.; Song, J.; Shi, J.; Morton, M.; Rajca, S.; Rajca, A.; Wang, H. J. Am. Chem. Soc. 2016, 138, 10002.

[7] (a) Tian, Y.; Wang, G.; Ma, Z.; Xu, L., Wang, H. Chem.-Eur. J. 2018, 24, 15993.

(b) Takeda, T.; Ozawa, M.; Akutagawa, T. Cryst. Growth Des. 2019, 19, 4784.

[8] Lai, X.; Meng, F.; Zhang, Q.; Wang, K.; Li, G.; Wen, Y.; Ma, H.; Li, W.; Li, X.; Kyaw, A.; Wang, K.; Sun, W.; Du, M.; Guo, X.; Wang,
J.; Huang, W. Sol. RRL 2019, 1900011.

[9] Zhang, W.; Xu L.; Song, J.; Ma, Z.; Wang, H. Chin. J. Org. Chem. 2018, 38, 1119 (in Chinese)

(张卫杰，徐莉，宋金生，马志英，王华，有机化学， 2018，38， 1119.)

[10] Zhao, Z.; Zheng, X.; Du, L.; Xiong, Y.; He, W.; Gao, X.; Li, C.; Liu, Y.; Xu, B.; Zhang, J.; Song, F.; Yu, Y.; Zhao, X.; Cai, Y.; He, X.; Kwok, R. T. K.; Lam, J. W. Y.; Huang, X.; Phillips, D. L.; Wang, H.; Tang, B. Nat. Commun. 2019, 10, 2952.

[11] Wang, Y.; Wang, Z.; Zhao, D.; Wang, Z.; Cheng, Y.; Wang, H. Synlett 2007, 2390.

[12] Yang, Y.; Gu, Y.; Ma, Z.; Xu, L.; Wang, K.; Zou, B.; Wang, H. Dye Pigm. 2020, in revision.

[13] Crosby, G. A.; Demas, J. N. J. Phys. Chem. 1971, 75, 991

[14] Mei, J.; Leung, N. L. C.; Kwok, R. T. K.; Lam, J. W. Y.; Tang, B. Chem. Rev. 2015, 115, 11718.

[15] Winnik, F. Chem. Rev. 1993, 93, 587.

[16] Dong, Y.; Xu, B.; Zhang, J.; Tan, X.; Wang, L.; Chen, J.; Lv, H.; Wen, S.; Li, B.; Ye, L.; Zou, B.; Tian, W. Angew. Chem., Int. Ed. 2012, 51, 10782 .

(Li, L.; Fan, Y.) 